\title{
Long-term economic impacts of exome sequencing for suspected monogenic disorders: diagnosis, management, and reproductive outcomes
}

\author{
Deborah Schofield, PhD (10) ${ }^{1}$, Luke Rynehart, BEcon (1) ${ }^{1}$, Rupendra Shresthra, PhD (1) ${ }^{1}$, \\ Susan M. White, $\mathrm{MBBS}^{2,3,4}$ and Zornitza Stark, BMBCh DM (i) ${ }^{2,3,4}$
}

Purpose: To undertake the first end-to-end cost-effectiveness analysis of exome sequencing (ES) in rare disease diagnosis.

Methods: A cohort of 80 infants who underwent ES and usual diagnostic care in parallel were used to model incremental cost and health outcomes (quality adjusted life-years, QALYs) attributable to ES diagnosis over a 20-year horizon. Three models were developed: (1) outcomes in patients only, (2) outcomes in patients and firstdegree relatives as a result of cascade testing, and (3) outcomes in patients and first-degree relatives including parental reproductive outcomes.

Results: When the directly observed cost and health outcomes of the cohort participants were projected, the use of ES resulted in a gain of 7.39 QALYs and an incremental cost-effectiveness ratio (ICER) of AU\$31,144.35 (i.e., cost per additional QALY gained).
When cascade testing in first-degree relatives was added, costeffectiveness increased, to a gain of 11.62 QALYs and an ICER of AU\$20,839.57. When parental reproductive outcomes were added, cost-effectiveness increased again, with 36.00 QALYs gained and an ICER of AU\$14,235.28.

Conclusion: Use of ES in suspected monogenic disorders becomes increasingly cost-effective as the benefits of ES data reanalysis, cascade testing in first-degree relatives, and parental reproductive outcomes are incorporated into modeling.

Genetics in Medicine (2019) 21:2586-2593; https://doi.org/10.1038/s41436019-0534-x

Keywords: exome sequencing; cost-effectiveness; QALY; ICER

\section{INTRODUCTION}

There is now substantial evidence that genomic tests such as exome and genome sequencing (ES/GS) are of high diagnostic and clinical utility in suspected pediatric rare genetic disease, with a recent meta-analysis of 37 studies, comprising 20,068 children, indicating a three- to fourfold improvement in diagnostic yield over the current accepted and funded firsttier test, chromosomal microarray. ${ }^{1}$ However, most studies to date have reported short-term outcomes of testing, such as immediate changes in usual diagnostic care and management, and very few have attempted to collect longer-term outcome data. $^{2-4}$

Furthermore, despite the increasing evidence of diagnostic and clinical utility, there are very few studies of the costeffectiveness of the use of ES/GS for the diagnosis of suspected monogenic disorders ${ }^{5,6}$ and fewer still have utilized outcome measures recommended for economic evaluations by major health technology assessment agencies such as survival or quality adjusted life-years (QALYs). Systematic investigation of the long-term clinical and cost impacts of genomic sequencing is needed to guide its implementation into health systems and services. Health system funders, in particular, need evidence for policy, resource allocation, and service planning.

Whilst cost-effectiveness analysis can use a variety of outcomes as a measure of effectiveness, such as prolongation of life or adverse events avoided, the QALY combines an assessment of longevity and quality of life outcomes that can be attributed to a particular intervention. The QALY is therefore the preferred metric of cost-effectiveness analysis because it is the most holistic measure of intervention effects, and is measured on a $0-1.00$ scale that allows for comparison across various interventions, allowing for analysis of the cost of financial resources across competing therapeutic areas within a health system budget. ${ }^{7}$ Whilst the Australian Pharmaceutical Benefits Advisory Committee (PBAC) does not employ a specific threshold, evidence suggests therapies are more likely to be reimbursed with a cost per QALY gained of around AU $\$ 30,000$ or less. ${ }^{8}$

\footnotetext{
${ }^{1}$ GenIMPACT: Centre for Economic Impacts of Genomic Medicine, Department of Economics, Faculty of Business and Economics, Macquarie University, Sydney, NSW, Australia ${ }^{2}$ Victorian Clinical Genetics Services, Murdoch Children's Research Institute, Melbourne, Australia; ${ }^{3}$ Department of Paediatrics, University of Melbourne, Melbourne, Australia; ${ }^{4}$ Melbourne Genomics Health Alliance, Melbourne, Australia. Correspondence: Luke Rynehart (luke.rynehart@mq.edu.au)
} 
A recent systematic literature review ${ }^{3}$ reported eight full economic evaluations in ES/GS studies, of which only two incorporated cost-utility analysis (QALYs), one in relation to secondary findings from next-generation sequencing (NGS) ${ }^{9}$ and the other in relation to bacterial genomic sequencing. ${ }^{10}$ Neither study is based on primary evidence found in prospective clinical studies and neither applies to rare genetic disease diagnosis.

We previously reported the first cost-effectiveness analysis of singleton ES compared with standard diagnostic care in a prospective clinical cohort of infants suspected of having monogenic disorders. Like many studies, we initially only reported cost-effectiveness in relation to cost per diagnosis as a short-term measure. Our modeling data suggested a potential saving of AU\$2182 (US\$1702) per additional diagnosis compared with the standard diagnostic pathway if ES is used as a first-tier sequencing test. ${ }^{5}$ A number of other studies using retrospective or hypothetical cohorts of patients with rare genetic disease have produced evidence supporting the early use of genomic testing in rare disease to improve cost-effectiveness of ES, but have similarly focused on the cost per diagnosis and not taken account of health outcomes. ${ }^{11-15}$

We recently reported health outcomes related to the use of ES for rare disease diagnosis using QALYs in the same cohort, ${ }^{6}$ including a cost saving of AU\$1578 per QALY gained for the probands. Taking account of ES data reanalysis, cascade testing, and reproductive service use by first-degree relatives at 18 months, there was an additional cost of $\mathrm{AU}$ $\$ 8118$ per QALY gained due to genomic sequencing.

This study builds on our previous analyses to model the incremental cost-effectiveness of ES in this prospectively ascertained cohort of infants with suspected monogenic disorders by projecting over a 20 -year time horizon. We combine this projection together with our previously published data to produce the first end-to-end costeffectiveness analysis of ES in rare disease diagnosis, both in the patients tested alone, and in their first-degree relatives.

\section{Study design}

\section{MATERIALS AND METHODS}

Infants with suspected monogenic disorders were recruited from a single tertiary pediatric center (Royal Children's Hospital, Melbourne, Australia) as part of a study undertaken by the Melbourne Genomics Health Alliance. The study was part of the Melbourne Genomics Health Alliance demonstration project (http://www.melbournegenomics.org.au) and received Human Research Ethics Committee approval (13/ $\mathrm{MH} / 326)$. Informed written consent was obtained from the parents of the participants.

Recruitment was prospective and is fully described elsewhere. ${ }^{5,6}$ Briefly, infants aged $0-2$ years were eligible if presenting with multiple congenital abnormalities and dysmorphic features or other features strongly suggestive of monogenic disorders, for example, neurometabolic conditions and skeletal dysplasias. Singleton ES was performed early in the diagnostic trajectory as a first-tier sequencing test in parallel with usual diagnostic care to develop counterfactual models measuring the incremental cost differences of ES versus standard care. Singleton ES was chosen for this study in preference to NGS panel, or trio ES or GS, to balance reduced sequencing costs (in comparison with trios) with the opportunity to expand the genes analyzed based on evolving phenotype information, and to reanalyze data in response to new gene discoveries.

Information on all diagnostic investigations, including those planned, was collected from referring clinicians and from medical records. Analysis of ES data was limited to genes known to cause monogenic disorders (the Mendeliome original size 2830 genes, current size 3842 genes; publicly available at https://www.vcgs. org.au/sites/default/files/downloads/TGW024_genelist_V3.pdf) and only variants relevant to the participant's phenotype were assessed with regard to pathogenicity. We collected information on continuing diagnostic investigation (including ES data reanalysis), changes in management, cascade testing, and parental reproductive outcomes in this cohort for a median duration of 473 days postresult as previously described. ${ }^{6}$

Specific health utility values for each child's health state were assigned based on parent-reported preferences for health states using utility values derived from a 2009 pediatric health utility study. ${ }^{16}$ Where possible we used patient reported survey data to assign a health state to a child, with clinical opinion being utilized when this was not available.

\section{Health economic analysis Diagnostic pathway costs}

Stark et al. ${ }^{5}$ examined three counterfactual models for the purpose of assessing the cost-effectiveness of incorporating ES at different time points in the diagnostic trajectory. In model 1, ES was used as a last resort test performed after all other diagnostic tests, including both actual and planned singlegene and multigene panel tests, are exhausted; in model 2, ES replaced single- and multigene panel tests and complex/ invasive tests; and in model 3, ES was used as a first-tier sequencing test replacing all other tests apart from chromosomal microarray. For the purpose of the current study, we have used model 2 because it most closely approximates likely clinical practice upon introduction of ES as an early test. In this model, patients undergo basic biochemical, imaging, and neurophysiological studies and subspecialist assessments, as in the standard diagnostic pathway, because these can guide empiric management while a definitive diagnosis is awaited. ES replaces all genetic testing except single-nucleotide polymorphism microarray analysis because the ES assay used was not accredited to detect structural variation. In this model, ES also replaces complex biochemistry testing, which is performed in specialized laboratories, and testing that is burdensome for patients, such as invasive tissue biopsies. Costs of sample shipments and of operating room/anesthetic time are eliminated as a result. There are two consultations with a clinical geneticist and genetic counselor per patient: one for initial assessment and ES initiation and the other for return of results. 


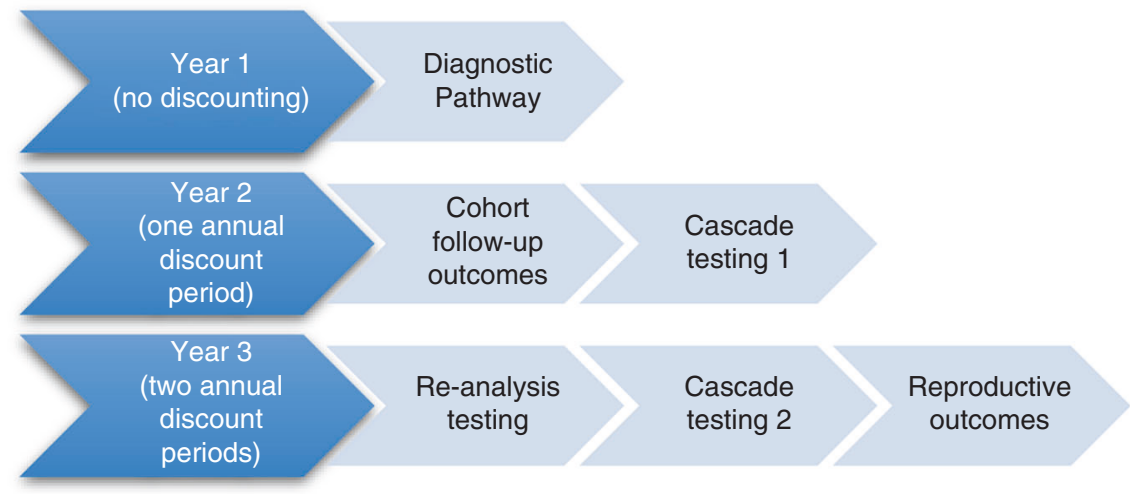

Fig. 1 Timing of Model Inputs

The observed costs by Stark et al. ${ }^{5}$ were revised to increase the cost of ES from AU\$2000.00 to AU $\$ 3100.00$ (current price from Victorian Clinical Genetic Services). ${ }^{17}$ This revision increases the average cost of the ES diagnostic pathway from AU $\$ 5913.79$ to AU $\$ 7013.79$ per patient. The average cost of the standard diagnostic pathway remains at AU\$4733.81, which increases the average incremental cost of performing ES from AU\$1179.98 to AU\$2279.98 per patient, and a total cost of AU $\$ 182,398.16$ for the cohort.

The cost of singleton ES currently varies between AU $\$ 1500$ (South Eastern Area Laboratory Services, personal communication) and AU\$3100 (Victorian Clinical Genetics Services) in Australian laboratories. There is wide variation in the cost of genomic testing internationally as well as in analysis and reporting practices, which makes comparisons difficult, but averages of USD\$4859 (AU\$6425.78) for trio exome sequencing (ES) and USD\$1944 (AU\$2570.84) for singleton ES have been reported in a recent benchmarking study. ${ }^{18}$

In sensitivity analysis we performed bootstrap simulations in SAS version 9.4 with 5000 replications to estimate total costs and QALYs in a cohort of 80 at the high/low Australian price-points, original price-point of AU\$2000, and the average cost of singleton ES reported for the United States.

\section{Projection methodology}

Due to the uncertainty in outcomes of rare genetic diseases diagnosed in this cohort, the projection is set at 20 years. This timeframe is considered adequate to examine the long-term cost-effectiveness of ES, without projecting beyond reasonable uncertainty.

This projection length differs from PBAC guidelines, which advise that when a treatment is expected to affect mortality or long-term outcomes, a lifetime projection is appropriate. ${ }^{19}$ Acknowledging this, extrapolations of up to 60 years are explored in sensitivity analysis. A base case annual discount ratio of $5 \%$ is applied, with sensitivity analysis of 3.5 and $0 \%$, in accordance with the PBAC guidelines. ${ }^{19}$

No deaths were assumed to occur as a result of syndromes within the projections (apart from the observed death of patient 204313). Overall survival probabilities were applied to all projected cost and health outcomes using the latest Australian life-table data. ${ }^{20}$

Population norm utility values were utilized to provide the most accurate estimation of incremental utility gained by ES. Assessment of quality of life (AQoL) utility norms were sourced from a 2013 study on the Australian population. The youngest age bracket was 16 to 19 years of age with a utility norm of 0.87 (ref. ${ }^{21}$ ). In absence of detailed utility norms for children aged under 16 , this utility norm of 0.87 is applied to ages 0 to 16 .

To apply age-specific overall survival probabilities and utility norms, the cohort members are assumed to be 2 years of age at the end of the follow-up period. The average age of parents at the time of childbirth was found to be 33.1 and 30.7 for fathers and mothers respectively, ${ }^{22}$ which was weighted to 32 using Australian demographic statistics. ${ }^{23}$

Diagnostic costs are assumed to occur within the first year of the model, at which time the patient is categorized as diagnosed by ES, diagnosed by standard diagnostic care, or undiagnosed. Using this assumption, diagnostic costs occur prior to any annual discounting, whilst all costs and health outcomes recorded during the follow-up period were subject to one period of annual discounting compared with the costs measured by Stark et al. ${ }^{6}$

Reanalysis is assumed to be performed once at 18 months after an initial noninformative ES outcome (third year of the model).

Cascade outcomes were included at two time points: the outcomes deriving from the patients who were diagnosed on initial ES testing (beginning of second year), and the cascade outcomes of the patients diagnosed by reanalysis of ES data (third year of the model).

Reproductive costs and outcomes were assumed to occur at the beginning of the third year (Fig. 1).

\section{RESULTS}

Of the 80 patients, a total of 48 received a diagnosis; 26 of these were diagnosed by ES only (4 of whom were diagnosed by reanalysis of ES data). A total of 22 diagnoses were achieved via the standard diagnostic pathway, inclusive of those who would have been diagnosed had the next planned 


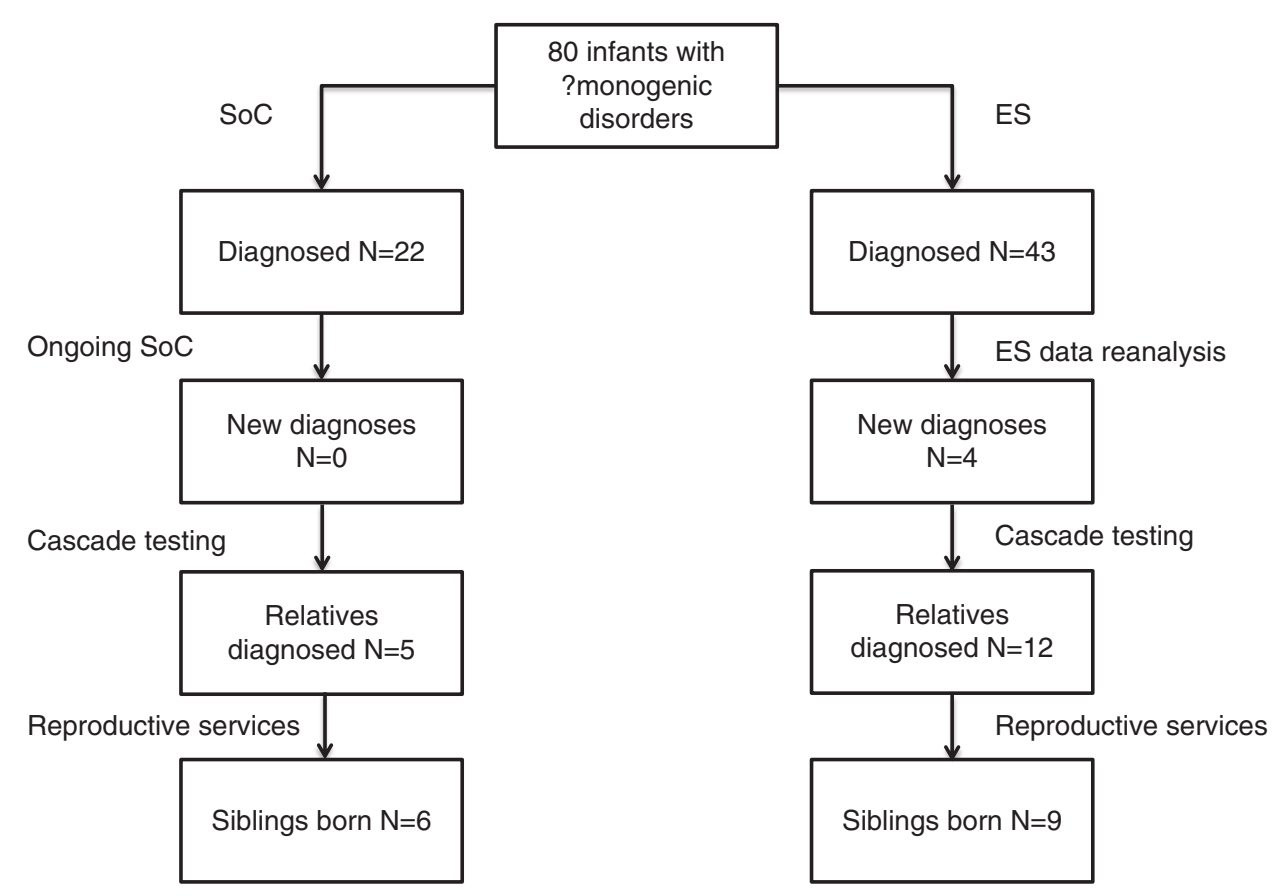

Fig. 2 Diagnoses and Reproductive Outcomes

test been undertaken. One diagnosis was made by standard diagnostics only (not ES).

Cascade testing was offered to all parents to clarify reproductive risks, and to siblings where clinically indicated. Seventy-nine of 88 eligible first-degree relatives underwent cascade testing. Twelve relatives of the infants diagnosed by ES received a molecular diagnosis following cascade testing. Two of these relatives had a change in management as a result.

A total of 16 couples sought advice from reproductive genetic services: 2 couples with undiagnosed children and 14 with diagnosed children. A total of nine pregnancies were recorded in the diagnosed group. Of these, three were diagnosed by ES only ${ }^{6}$ (Fig. 2).

\section{Cost-effectiveness analysis}

Model 1: cost and health outcomes in probands only

Cohort outcomes prior to reanalysis of ES data. Using ES to replace single- and multigene panel tests and complex/invasive tests was found to come at a cost of AU\$182,398.16 above the standard diagnostic pathway. Of the 80 patients, 10 infants incurred additional cost to the health system due to ES diagnosis during follow-up, with 6 of these infants assumed to have ongoing additional costs beyond follow-up. These additional costs for the cohort were AU $\$ 51,465.65$ when projected over 20 years.

Three patients were recorded as experiencing altered health outcomes as a result of ES diagnosis. ${ }^{6}$

Patient 204313 was diagnosed with combined oxidative phosphorylation deficiency 11 (RMND1) and experienced life prolongation of 12 months at severe disability for a QALY gain of 0.59 (discounted to 0.56). Additional details on QALY projections can be found in Table S1.

Patient 204405 had received an average of 2.5 blood transfusions over the previous 2 years, prior to starting treatment with prednisolone as a result of ES diagnosis. Conservatively, it is expected that at least one blood transfusion per year would have continued in absence of the ES diagnosis. The ongoing need for transfusions is estimated to result in a disutility of 0.06 (ref. ${ }^{6}$ ). The model therefore assumes a 0.06 QALY gain for each of the projected years, resulting in a total QALY gain of 0.72 .

Patient 204320 was diagnosed with thiamine transporter dysfunction syndrome, due to compound heterozygous pathogenic variations in SLC19A3. The patient experienced onset of symptoms at 5 months, and progressed to a severe level of disability (survey-reported health utility of 0.60 ) at the age of 15 months, at which point the symptoms were stabilized. A literature review of published thiamine transporter dysfunction syndrome case studies indicates that symptoms tend to worsen over time and stabilize at their present level of disability upon diagnosis and treatment. ${ }^{24-32}$

Based on the clinical trajectory prior to diagnosis, we estimate that without ES diagnosis, death would have occurred very rapidly at 41 months of age (see Table S1).

The projected outcomes for this patient result in 6.11 QALYs gained as a result of ES diagnosis.

Sensitivity analysis is performed in which the patient may have received a diagnosis via further standard diagnostics at a later time.

Cohort outcomes of ES data reanalysis. The total cost of performing ES data reanalysis on unsolved cases with ongoing suspicion of monogenic disorder at 18 months was $\mathrm{AU}$ 
Table 1 Cost-effectiveness outcomes (\$AU)

\begin{tabular}{|c|c|c|c|c|c|}
\hline Stage & Cost of stage & $\begin{array}{l}\text { QALY gain } \\
\text { per stage }\end{array}$ & Cumulative cost & $\begin{array}{l}\text { Cumulative } \\
\text { QALY gain }\end{array}$ & Cumulative ICER \\
\hline Cohort outcomes & $\$ 230,226.67$ & 7.39 & $\$ 230,226.67$ & 7.39 & $\$ 31,144.35$ \\
\hline $\begin{array}{l}\text { Cohort + cascade + reproductive } \\
\text { outcomes }\end{array}$ & $\$ 270,351.42$ & 24.38 & $\$ 512,505.59$ & 36.00 & $\$ 14,235.28$ \\
\hline
\end{tabular}

ICER incremental cost-effectiveness ratio, QALY quality adjusted life-year.

$\$ 11,350.00$. This represents a cost saving of AU\$4234.94 compared with the ongoing standard diagnostic care costs of $\$ 15,584.94$, which occurred in parallel in this cohort per the study design. One patient received a renal ultrasound as a result of reanalysis diagnosis, at an additional cost of $\mathrm{AU}$ $\$ 225.00$ (ref. $^{6}$ ).

Reanalysis was modeled to occur in the third year of the model, resulting in a total discounted cost saving of $\mathrm{AU}$ $\$ 3637.13$.

Adding the cost savings attributable to reanalysis of ES data to the projected costs of the patients who were diagnosed prior to reanalysis results in a total projected cost of $\mathrm{AU}$ $\$ 47,828.51$.

Total costs and health outcomes for the cohort. Combining the projected cost of outcomes of the cohort (AU\$47,828.51, inclusive of reanalysis) with the costs of the diagnostic pathway (AU\$182,398.16) results in a cumulative cost of $\mathrm{AU}$ $\$ 230,226.67$, and a total QALY gain of 7.39 , resulting in an incremental cost-effectiveness ratio (ICER) of AU\$31,144.35 per QALY gained (Table 1).

\section{Model 2: cost and health outcomes in probands and first- degree relatives}

Cascade testing was offered to all parents to clarify reproductive risks, and to siblings where clinically indicated. At follow-up, cascade testing was found to come at a total cost of AU\$28,000 for the entire cohort, and an average cost of AU\$583.33 per diagnosed patient. ${ }^{6}$ Of the 26 patients diagnosed by ES only, 22 were diagnosed on first analysis, and the remaining 4 on reanalysis, resulting in discounted costs of AU\$555.56 each for those diagnosed on first analysis, and discounted costs of AU\$529.10 each for those diagnosed on reanalysis. The total discounted cost of cascade testing due to the additional diagnoses made by ES was AU\$14,338.62.

Two additional diagnoses were achieved as a result of ES proband diagnosis, both with ongoing health/cost outcomes.

A parent was started on a cardiac surveillance program that is estimated to come at a total cost of AU\$3895.06 for the 20year projection.

Additionally, an asymptomatic sibling of patient 204320 was diagnosed with thiamine transporter dysfunction syndrome via cascade testing and commenced on treatment prior to experiencing symptoms. The diagnosed sibling was 4 years older than the proband.

To model the counterfactual scenario that would have occurred without therapy, analysis of the symptom onset and genotype-phenotype relationship of the condition was undertaken.

A systematic review of thiamine transporter-2 deficiency case studies found the mean average age of symptom onset to be 3.5 years, with $80 \%$ of the 69 patients within the review experiencing symptom onset before the age of 12 (ref. ${ }^{30}$ ).

From this we assume that it is most likely the asymptomatic sibling in our study would have developed symptoms within childhood without prophylactic therapy. We therefore build the base case counterfactual model assuming the sibling would have experienced the same neurological deterioration observed in the proband, beginning at 12 years of age.

Acknowledging the paucity of evidence regarding genotype-phenotype relationships, a sensitivity analysis is performed in which the sibling would never have experienced symptom onset, and therefore no health outcomes are attributed to the prophylactic therapy.

In our base case scenario, the prophylactic treatment of the sibling comes at a cost saving of AU\$6306.18 due to hospitalizations and health resources avoided, and a total QALY gain of 4.23.

Adding the projected costs and health outcomes from cascade testing to the outcomes of the cohort resulted in a cumulative cost of AU\$242,154.17, and a cumulative QALY gain of 11.62, resulting in an ICER of AU\$20,839.57 per QALY gained.

\section{Model 3: cost and health outcomes of probands, first-} degree relatives, and parental reproductive outcomes Three babies were born among the 26 families diagnosed by ES only. One baby was born among the 32 families with no diagnosis, resulting in a birth rate of $3.13 \%$. From this we assume that $0.8125(26 \times 3.13 \%)$ births would have resulted among the 26 families diagnosed by ES if no diagnosis had been made, resulting in 2.1875 additional births attributable to ES. We therefore attribute $72.92 \%(2.1875 / 3)$ of all projected costs and outcomes for the three recorded births to the ES diagnosis.

One affected child was born in the ES diagnosis group. The couple chose to continue an affected 
pregnancy after prenatal diagnosis because the condition is potentially curable by bone marrow transplantation (BMT).

The combined cost of reproductive outcomes for families diagnosed by ES only are AU $\$ 270,351.42$. See Table S2 for details.

Conservatively, the BMT is assumed to result in a utility state of 0.78 for the entire first year of life using the utility state for "Survivors of pediatric intensive care for unspecified reasons" derived by Kwon using Health Utilities Index (HUI2, Table 3), ${ }^{33}$ with population norm utility assumed for the remainder of the projection.

Both of the remaining births were unaffected and are assumed to remain at population norm utility.

Additionally, both parents were assumed to benefit from a utility gain of 0.07 each as a result of the birth of their additional child based on the utility gained as a result of fertility as reported for the Health Utilities Index (HUI2). ${ }^{34}$

Adjusting for the incremental birth rate of ES diagnosis, and applying overall survival probabilities and annual discounting results in a total projected QALY gain of 24.38 for reproductive outcomes.

Adding the projected parental reproductive costs and outcomes attributable to ES diagnosis to those of the proband and first-degree relatives results in a cumulative cost of $\mathrm{AU}$ $\$ 512,505.59$, and a cumulative QALY gain of 36.00 , resulting in an ICER of AU\$14,235.28 per QALY gained.

\section{Sensitivity}

Length of projection period and discount rates

When projection length is extended, or discount rates reduced, ES becomes increasingly cost-effective (Table 2).

\section{Bootstrapping and ES price sensitivity}

The lower price for ES makes its use considerably more costeffective, with the ICER for cohort, cascade, and reproductive outcomes being AU\$10,654.20 per QALY gained at the lowest price (AU $\$ 1500$ ), and AU $\$ 13,029.23$ at the average US price (AU\$2570.84) (Table 3).

\section{Proband delayed diagnosis}

In the unlikely event that patient 204320, who had received an incorrect diagnosis following magnetic resonance imaging (MRI), had a further MRI 18 months after symptom onset and received a correct diagnosis (see Table S1), ICERs from the 20-year projection are $\$ 54,320.83, \$ 26,239.47$, and $\$ 14,744.50$, for models 1,2 , and 3 respectively.

\section{Asymptomatic sibling}

If we do not attribute any health outcomes to the asymptomatic sibling of patient 204320, the prophylactic treatment is found to come at a total cost of AU $\$ 1779.93$ in the 20-year projection. In this scenario ICERs are $\$ 31,144.35$, $\$ 33,851.73$, and $\$ 16,383.78$, for models 1,2 , and 3 respectively.

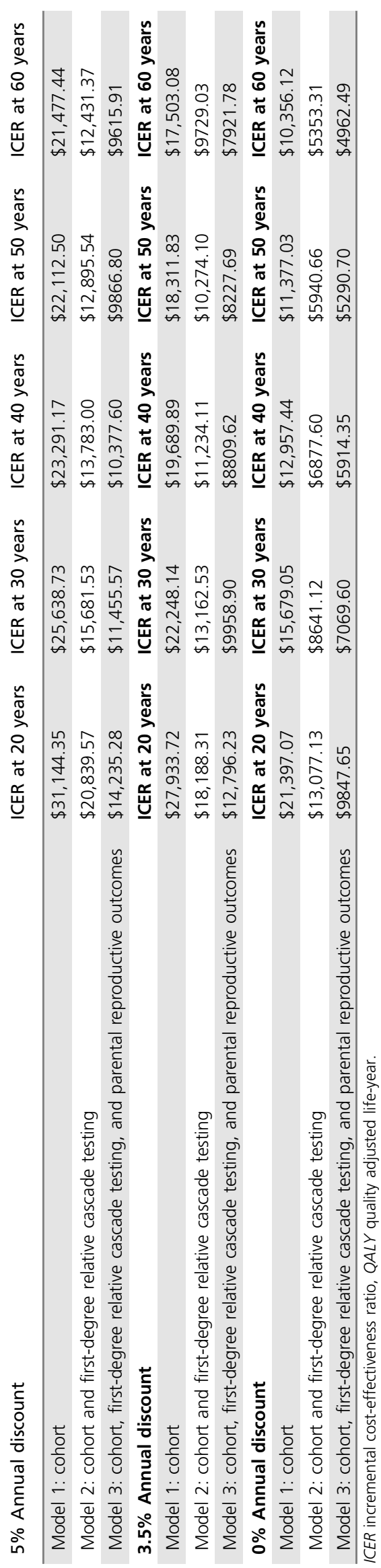


Table 3 Sensitivity: cost of ES (\$AU)

\begin{tabular}{|c|c|c|c|}
\hline Price of ES & Costs & QALYs & ICER \\
\hline \multicolumn{4}{|c|}{ Model 1: cohort outcomes } \\
\hline$\$ 3100$ (Base model) & $\$ 230,429.34$ & 7.43 & $\$ 31,013.37$ \\
\hline$\$ 1500$ & $\$ 102,429.34$ & 7.43 & $\$ 13,785.91$ \\
\hline$\$ 2000$ & $\$ 142,429.34$ & 7.43 & $\$ 19,169.49$ \\
\hline$\$ 2570.84$ & $\$ 188,096.54$ & 7.43 & $\$ 25,315.82$ \\
\hline \multicolumn{4}{|c|}{ Model 2: cohort and cascade testing of first-degree relatives } \\
\hline$\$ 3100$ (Base model) & $\$ 242,269.97$ & 11.69 & $\$ 20,724.55$ \\
\hline$\$ 1500$ & $\$ 114,269.97$ & 11.69 & $\$ 9775.02$ \\
\hline$\$ 2000$ & $\$ 154,269.97$ & 11.69 & $\$ 13,196.75$ \\
\hline$\$ 2570.84$ & $\$ 199,937.17$ & 11.69 & $\$ 17,103.27$ \\
\hline \multicolumn{4}{|c|}{ Model 3: cohort, cascade, and parental reproductive outcomes } \\
\hline$\$ 3100$ (Base model) & $\$ 512,296.95$ & 36.07 & $\$ 14,202.85$ \\
\hline$\$ 1500$ & $\$ 384,296.95$ & 36.07 & $\$ 10,654.20$ \\
\hline$\$ 2000$ & $\$ 424,296.95$ & 36.07 & $\$ 11,763.15$ \\
\hline$\$ 2570.84$ & $\$ 469,964.15$ & 36.07 & $\$ 13,029.23$ \\
\hline
\end{tabular}

ES exome sequencing, ICER incremental cost-effectiveness ratio, QALY quality adjusted life-year.

\section{DISCUSSION}

Genomic testing is a transformative technology and a growing body of research anticipates it to have a major effect on rare disease diagnosis and pediatric care. ${ }^{35,36}$

Despite being based on a clinical cohort, the study has some limitations, particularly the need to make assumptions, although based on available literature, about long-term patient outcomes. Nonetheless, the outcomes are conservative in using the highest current price for ES and selecting a 20year projection rather than a longer period in our base case. The projection is also conservative in only modeling the health outcomes of parental reproductive outcomes that occurred within the follow-up period despite the fact that parents may continue to have additional healthy children due to ES diagnosis. The projection is also conservative in the estimation of further diagnostic costs in the standard diagnostic pathway. It is also likely that ongoing reanalysis of genomic data in light of new gene discoveries and improved bioinformatics pipelines will continue to yield more diagnoses in the future, ${ }^{37,38}$ although it is currently unclear if this may plateau as the pace of gene discovery slows. Despite this, the model returns a very cost-effective ICER of AU\$31,144.35 when only accounting for the outcomes of the cohort, improving to AU\$20,839.57 and AU\$14,235.28 as the benefits in cascade-tested first-degree relatives and parental reproductive outcomes are added to the model. It is important to note that this is a prospectively ascertained cohort of infants, who received genomic testing very early in the diagnostic trajectory as a first-tier sequencing test. Many of the health and economic benefits of early diagnosis, such as avoidance of exhaustive investigation, opportunities to influence management, and providing timely reproductive advice are unlikely to be applicable if genomic testing is used as a test of last resort later in the diagnostic trajectory.
This study has provided some important lessons in terms of the development of robust economic studies in the field of genomics. Firstly, clinical genetics is focused on treating families, not just individuals, and thus it is critical that a comprehensive cost-effectiveness study takes into account both cascade testing and reproductive costs and health impacts, which in our study resulted in a more costeffective outcome. Secondly, capturing health outcomes using standard measures such as quality adjusted life-years gained (QALYs) is essential for comparing the cost-effectiveness of genomic testing with other types of testing or health investment. As Sagoo et al. ${ }^{39}$ note, whilst there exist utilitybased willingness-to-pay thresholds for government funding, there is no universally acknowledged willingness-to-pay threshold for a diagnosis alone. Thirdly, comprehensive studies take account of long-term outcomes. This is important but also challenging in the case of rare diseases where there is often scant data on long-term patient outcomes, particularly for conditions for which the molecular basis of the disease has only very recently been elucidated. Accordingly, sensitivity analysis may be necessary to assess the impact of a range of plausible outcomes. Finally, as this field emerges, the rigor of analyses will improve as economists and clinicians working in genomics collaborate together, thereby developing the economic literacy of clinical geneticists and the genomic literacy of economists.

\section{SUPPLEMENTARY INFORMATION}

The online version of this article (https://doi.org/10.1038/s41436019-0534-x) contains supplementary material, which is available to authorized users.

\section{DISCLOSURE}

The authors declare no conflicts of interest.

Publisher's note: Springer Nature remains neutral with regard to jurisdictional claims in published maps and institutional affiliations.

\section{REFERENCES}

1. Clark MM, Stark Z, Farnaes L, et al. Meta-analysis of the diagnostic and clinical utility of genome and exome sequencing and chromosomal microarray in children with suspected genetic diseases. NPJ Genom Med. 2018;3:16.

2. Hayeems RZ, Bhawra J, Tsiplova K, et al. Care and cost consequences of pediatric whole genome sequencing compared to chromosome microarray. Eur J Hum Genet. 2017;25:1303-1312.

3. Schwarze K, Buchanan J, Taylor JC, Wordsworth S. Are whole-exome and whole-genome sequencing approaches cost-effective? A systematic review of the literature. Genet Med. 2018;20:112-1130.

4. Vrijenhoek T, Middelburg EM, Monroe GR, et al. Whole-exome sequencing in intellectual disability; cost before and after a diagnosis. Eur J Hum Genet. 2018;26:1566-1571.

5. Stark Z, Schofield D, Alam K, et al. Prospective comparison of the costeffectiveness of clinical whole-exome sequencing with that of usual care overwhelmingly supports early use and reimbursement. Genet Med. 2017:19:867-874.

6. Stark Z, Schofield D, Martyn M, et al. Does genomic sequencing early in the diagnostic trajectory make a difference? A follow up study of clinical effectiveness. Genet Med. 2019;21:173-180. 
7. Drummond MF, Sculpher MJ, Claxton K, Stoddart GL, Torrance GW. Methods for economic evaluation of health care programmes. Oxford: Oxford University Press; 2015.

8. Access to Medicines Working Group: Australian Government Department of Health. The effects of statutory price reductions on the listing of new medicines. 2008. http://www.pbs.gov.au/info/general/ working-groups/amwg/amwg-interim-report-attachment-a. Accessed 1 January 2019

9. Bennette CSGC, Burke W, Jarvik GP, Veenstra DL. The cost-effectiveness of returning incidental findings from next-generation genomic sequencing. Genet Med. 2015;17:587-595.

10. Buchanan-Hughes AMGA, Evans J, Slater D, Eddowes LA. Investigating the cost-effectiveness of bacterial whole-genome sequencing for enabling targeted antibiotic selection In urinary tract infections. Value Health. 2015;18:A510.

11. Palmer EE, Schofield D, Shrestha $R$, et al. Integrating exome sequencing into a diagnostic pathway for epileptic encephalopathy: evidence of clinical utility and cost effectiveness. Mol Genet Genomic Med. 2018;6:186-199.

12. Shashi V, McConkie-Rosell A, Rosell B, et al. The utility of the traditional medical genetics diagnostic evaluation in the context of next-generation sequencing for undiagnosed genetic disorders. Genet Med. 2014;16:176-182

13. Soden SE, Saunders CJ, Willig LK, et al. Effectiveness of exome and genome sequencing guided by acuity of illness for diagnosis of neurodevelopmental disorders. Sci Transl Med. 2014;6:265ra168.

14. Tan TY, Dillon OJ, Stark $Z$, et al. Diagnostic impact and cost-effectiveness of whole-exome sequencing for ambulant children with suspected monogenic conditions. JAMA Pediatr. 2017;171:855-862.

15. van Nimwegen KJ, Schieving JH, Willemsen MA, et al. The diagnostic pathway in complex paediatric neurology: a cost analysis. Eur J Paediatr Neurol. 2015;19:233-239.

16. Carroll AE, Downs SM. Improving decision analyses: parent preferences (utility values) for pediatric health outcomes. J Pediatr. 2009;155:21-25. 25 e21-25

17. VCGS. Genomics price list. 2018. https://www.vcgs.org.au/node/1024. Accessed 18 September 2018.

18. Dragojlovic N, Elliott AM, Adam S, et al. The cost and diagnostic yield of exome sequencing for children with suspected genetic disorders: a benchmarking study. Genet Med. 2018;20:1013-1021.

19. Australian Government Department of Health. Guidelines for preparing submissions to the Pharmaceutical Benefits Advisory Committee (PBAC). http://www.pbac.pbs.gov.au. Accessed 18 September 2018.

20. Australian Bureau of Statistics. 3302.0.55.001-Life tables, states, territories and Australia, 2009-11. http://www.abs.gov.au/AUSSTATS/ abs@.nsf/Lookup/3302.0.55.001Main+Features12009-2011? OpenDocument. Accessed 1 February 2019

21. Hawthorne G, Korn S, Richardson J. Population norms for the AQoL derived from the 2007 Australian National Survey of Mental Health and Wellbeing. Aust N Z J Public Health. 2013;37:7-16.

22. Australian Bureau of Statistics. 3301.0-Births, Australia, 2007. http://www.abs.gov.au/AUSSTATS/abs@.nsf/Lookup/3301.0Main +Features12007? OpenDocument. Accessed 1 February 2019.

23. Australian Bureau of Statistics. 3101.0-Australian demographic statistics, Jun 2018. https://www.abs.gov.au/AUSSTATS/abs@.nsf/
Lookup/3101.0Main+Features1Jun\%202018?OpenDocument. Accessed 1 February 2019.

24. Alfadhel $M$, Almuntashri $M$, Jadah $R H$, et al. Biotin-responsive basal ganglia disease should be renamed biotin-thiamine-responsive basal ganglia disease: a retrospective review of the clinical, radiological and molecular findings of 18 new cases. Orphanet J Rare Dis. 2013;8:83.

25. Aljabri MF, Kamal NM, Arif M, AlQaedi AM, Santali EY. A case report of biotin-thiamine-responsive basal ganglia disease in a Saudi child: is extended genetic family study recommended? Medicine (Baltimore). 2016;95:e4819.

26. Debs R, Depienne C, Rastetter A, et al. Biotin-responsive basal ganglia disease in ethnic Europeans with novel SLC19A3 mutations. Arch Neurol. 2010;67:126-130.

27. Distelmaier $F$, Huppke $P$, Pieperhoff $P$, et al. Biotin-responsive basal ganglia disease: a treatable differential diagnosis of Leigh syndrome. JIMD Rep. 2014:13:53-57.

28. Flones I, Sztromwasser P, Haugarvoll K, et al. Novel SLC19A3 promoter deletion and allelic silencing in biotin-thiamine-responsive basal ganglia encephalopathy. PLoS ONE 2016;11:e0149055.

29. Kevelam SH, Bugiani M, Salomons GS, et al. Exome sequencing reveals mutated SLC19A3 in patients with an early-infantile, lethal encephalopathy. Brain. 2013;136 pt 5:1534-1543.

30. Ortigoza-Escobar JD, Serrano M, Molero M, et al. Thiamine transporter-2 deficiency: outcome and treatment monitoring. Orphanet J Rare Dis. 2014:9:92.

31. Serrano M, Rebollo M, Depienne C, et al. Reversible generalized dystonia and encephalopathy from thiamine transporter 2 deficiency. Mov Disord. 2012:27:1295-1298.

32. Yamada K, Miura K, Hara K, et al. A wide spectrum of clinical and brain MRI findings in patients with SLC19A3 mutations. BMC Med Genet. 2010;11:171.

33. Kwon J, Kim SW, Ungar WJ, Tsiplova K, Madan J, Petrou S. A systematic review and meta-analysis of childhood health utilities. Med Decis Making. 2018:38:277-305

34. Torrance GW, Feeny DH, Furlong WJ, Barr RD, Zhang Y, Wang Q. Multiattribute utility function for a comprehensive health status classification system. Health Utilities Index Mark 2. Med Care. 1996;34:702-722.

35. Doble B, Schofield DJ, Roscioli T, Mattick JS. Prioritising the application of genomic medicine. NPJ Genom Med. 2017;2:35.

36. Doble B, Schofield DJ, Roscioli T, Mattick JS. The promise of personalised medicine. Lancet. 2016;387:433-434.

37. Nambot $S$, Thevenon J, Kuentz $P$, et al. Clinical whole-exome sequencing for the diagnosis of rare disorders with congenital anomalies and/or intellectual disability: substantial interest of prospective annual reanalysis. Genet Med. 2018;20:645-654.

38. Wright CF, McRae JF, Clayton S, et al. Making new genetic diagnoses with old data: iterative reanalysis and reporting from genome-wide data in 1,133 families with developmental disorders. Genet Med. 2018;20:1216-1223.

39. Sagoo G, Norbury G, Mohammed S, Kroese M. Whole-exome sequencing in clinical genetics: a health economic evaluation. PHG Foundation; 2017. http://www.phgfoundation.org/report/whole-exomesequencing-clinical-genetics. Accessed 20 May 2019. 